\title{
Language context guides memory content
}

\author{
Viorica Marian and Margarita Kaushanskaya \\ Northwestern University, Evanston, Illinois
}

\begin{abstract}
The relationship between language and memory was examined by testing accessibility of general knowledge across two languages in bilinguals. Mandarin-English speakers were asked questions such as "name a statue of someone standing with a raised arm while looking into the distance" and were more likely to name the Statue of Liberty when asked in English and the Statue of Mao when asked in Mandarin. Multivalent information (i.e., multiple possible answers to a question) and bivalent information (i.e., two possible answers to a question) were more susceptible to language dependency than univalent information (i.e., one possible answer to a question). Accuracy of retrieval showed language-dependent memory effects in both languages, while speed of retrieval showed language-dependent memory effects only in bilinguals' more proficient language. These findings suggest that memory and language are tightly connected and that linguistic context at the time of learning may become integrated into memory content.
\end{abstract}

Language is known to guide conceptual development (Gopnik \& Meltzoff, 1997; Waxman \& Braun, 2005) and to provide a framework for mental representations (Gentner \& Goldin-Meadow, 2003; Gumperz \& Levinson 1996). Here we examine the relationship between language and memory by testing accessibility of general knowledge across two languages in bilinguals. Because bilinguals encode some of their memories while using one language and other memories while using the other language, bilingualism can, in essence, serve as a real-world laboratory for testing hypotheses about the interaction between memory and language.

Using a bilingual framework, we have recently proposed (Marian \& Neisser, 2000) that language context leads to encoding specificity effects (Davies \& Thomson, 1988; Tulving \& Thomson, 1973) and that linguistic factors at the time of recall may influence memory accessibility. The link between language and memory has emerged most consistently in autobiographical memory (Koven, 2001; Larsen, Schrauf, Fromholt, \& Rubin, 2002). For instance, the accessibility of autobiographical memories was improved when the language used at the time of remembering corresponded to the language in which memories were initially formed (Marian \& Neisser, 2000; Matsumoto \& Stanny, 2006; Schrauf \& Rubin, 1998). Language-dependent effects in autobiographical retrieval have been explained by the influence of a general linguistic milieu, as well as by language-specific wordprompt cuing (e.g., Marian \& Neisser, 2000; Matsumoto \& Stanny, 2006).

Recent findings suggest that language-dependency effects are found not only in episodic memory, but also in self-construal (e.g., Ross, Xun, \& Wilson, 2002) and in academic learning (e.g., Marian \& Fausey, 2006). For ex- ample, Ross et al. (2002) found that bilinguals differed in number of collective self-statements, self-esteem ratings, and cultural views when responding in their two languages. For academic learning, Marian and Fausey (2006) found that bilinguals were better at remembering information (e.g., about history, chemistry, etc.) when tested in the same language in which the material was originally learned (however, language proficiency modulated the effects). Moreover, other studies focused on performance in laboratory tasks such as learning of word lists. Retrieval of words encoded in mixed-language lists was found to cluster by language (e.g., Dalrymple-Alford \& Aamiry, 1969), and words that were learned and tested in the same language showed a retrieval advantage compared to words that were learned and tested in different languages (Durgunoğlu \& Roediger, 1987). However, it has been argued that studies of word lists and of memory for academic material, while informative in terms of memory organization, may not be indicative of memory performance in real-world environments (Koriat \& Goldsmith, 1996; Neisser, 1978). The question remains whether everyday semantic memory is susceptible to language influences. Demonstrating language-dependent recall for factual knowledge about the world would suggest that language effects permeate all aspects of declarative (Baddeley, 1982; Schacter \& Tulving, 1994) memory.

The present research examined the influence of language on accessibility of everyday knowledge. This study is the first to take an ecologically valid approach to semantic memory and to compare language-dependency across different types of knowledge. It was hypothesized that accessibility of general knowledge would be facilitated by congruence between languages at encoding and retrieval and that the magnitude of the effect would

V.Marian, v-marian@northwestern.edu 
vary across different types of knowledge. Specifically, it was hypothesized that responses that have more than one correct alternative would be more prone to languagedependent access, because both languages could have been used at encoding and because encoding language could serve as an additional variable influencing response selection. Examples of memory that is multivalent in nature include exemplars within a category (e.g., names of famous writers) and many autobiographical memories (e.g., a word prompt such as doctor could potentially elicit multiple alternatives). Examples of memory that is univalent in nature include academic material such as a chemical formula and factual information such as the name of the first American president. While multivalent and bivalent forms of knowledge can include information encoded in either of the bilinguals' two languages, univalent forms of knowledge include information encoded in only one of the bilinguals' two languages. The present study tested the hypothesis that susceptibility to language dependency varies across different types of knowledge by constructing questions that have either one, two, or more than two correct answers. It was predicted that the influence of language on semantic memory would be stronger for the bivalent and multivalent memory tasks than for the univalent memory task.

In the present study, accessibility of knowledge was measured using response accuracy (proportion of correct answers) and speed of retrieval (reaction time). Accuracy of retrieval reflected the nature of the retrieved memories, and speed of retrieval reflected efficiency of access to these memories. Prior research found that a match between languages of encoding and retrieval facilitated both the accuracy of memory retrieval, and the efficiency of memory retrieval (e.g., Marian \& Fausey, 2006; Marian \& Neisser, 2000). For instance, Marian and Fausey showed that material learned in the native language was retrieved faster when probed in L1 than when probed in L2. In the current study, it was predicted that both the nature and the efficiency of retrieved memories would be susceptible to language-dependency effects.

\section{METHOD}

\section{Participants}

Twenty Mandarin-English bilinguals were tested (mean age $=$ 32 years; $S E=2$ years; native language, Mandarin). All participants completed the Language Experience and Proficiency Questionnaire (LEAP-Q; Marian, Blumenfeld, \& Kaushanskaya, 2007), providing information about their language-learning history, language proficiency, and language use. Participants reported that they began learning English at an average age of 11.5 years $(S E=0.60)$, and had lived in an English-speaking country for an average of 5.5 years. Participants rated their speaking, reading, and understanding proficiency in English and in Mandarin on a scale from zero (no knowledge of a language) to five (native-speaker knowledge of a language). Comparison of proficiency levels in the two languages revealed that participants were more proficient speaking in Manda$\operatorname{rin}(M=4.8, S E=0.09$, range $=4-5)$ than in English $(M=3.35$, $S E=0.17$, range $=2-5)[t(19)=7.86, p<.0001]$, understanding Mandarin $(M=4.9, S E=0.07$, range $=4-5)$ than English $(M=$ $3.85, S E=0.15$, range $=2-5)[t(19)=6.84, p<.0001]$, and reading Mandarin $(M=4.85, S E=0.08$, range $=4-5)$ than English $(M=4.05, S E=0.13$, range $=3-5)[t(19)=5.14, p<.001]$.

\section{Materials}

Three tasks were used to test retrieval of general world knowledge in Mandarin-English bilinguals. Each of the three tasks included 16 questions: one set of 8 questions that were asked in Mandarin and one set of 8 questions that were asked in English. Questions were blocked by language, with order and the question set counterbalanced across participants.

The multivalent task tested retrieval of multiple items in a category. For instance, when prompted with "lakes" or with "famous actors," participants could name any four exemplars within those categories. Responses may have been learned in Mandarin (e.g., Qinghai Lake, Lake Poyang, Dongting Lake, Lake Tianchi, and Ge You, Liu Hua, Lu Yi, Chen Kun) or in English (e.g., Lake Michigan, Lake Ontario, Lake Erie, Lake Superior, and Cary Grant, Sidney Poitier, George Clooney, Kevin Costner).

The bivalent task tested responses to questions that had two possible correct answers, with each answer learned in either of the two languages. For instance, when asked "In a famous love story, what were the names of two lovers who died because of family disapproval?" participants could answer with either Romeo and Juliet or Liang Shanbo and Zhu Yingtai. Similarly, answers to questions such as "Where and when did Japan launch the initial attack during World War II?" and "Name a woman who succeeded in spite of a severe physical handicap" could differ depending on whether bilinguals drew from the body of knowledge acquired in English (Pearl Harbor, 1941 and Helen Keller) or from the body of knowledge acquired in Mandarin (Lugouqiao, 1937 and Zhang Haidi).

The univalent task tested retrieval of a single available correct answer, encoded in only one of the bilinguals' languages. For instance, when asked to name the mayor of Chicago, the only correct answer could be Richard Daley. ${ }^{1}$ Other examples of questions targeting material most likely encoded in English include "Name the capital of Illinois" and "Name the el-train line that connects Howard and 95th/Dan Ryan." Examples of questions targeting material most likely encoded in Mandarin include "Name the capital of the Sichuan province" and "Name the city used as a transfer from mainland China to Taiwan."

\section{Procedure}

A fluent bilingual experimenter tested participants individually, in English and in Mandarin, with language order counterbalanced across participants and tasks. While retrieval language was controlled experimentally, encoding language was manipulated via question design and by questioning participants at the end of the experiment. In general, it has been found that bilinguals are highly accurate in remembering whether information was presented in one or the other language (Kintsch, 1970). The research objective was disguised by telling participants that the study compared the number of questions answered correctly by bilinguals and monolinguals; when questioned at the end of the experimental session, none of the participants identified the actual objective of the study.

\section{Analyses}

The proportion of questions answered correctly and the reaction times were analyzed using $2 \times 2 \times 3$ ANOVAs, with language of encoding (English or Mandarin), language of retrieval (English or Mandarin), and task (multivalent, bivalent, or univalent) as withinsubjects variables. In the multivalent task, data were averaged across the four responses for the overall analyses; in addition, each of the four responses was also analyzed individually. Reaction times were calculated by coding digital recordings of the interview. A second independent rater coded $20 \%$ of all data, and point-to-point reliability between the two coders was $99.9 \%$ for accuracy and $88 \%$ for reaction time.

Additional analyses were performed to examine whether languagedependent memory effects varied with language proficiency. Two measures of language proficiency were used to divide participants into groups. Each grouping was entered as an independent between-subjects variable into exploratory analyses of variance. The first measure relied on self-reported speaking proficiency, be- 
cause speaking proficiency was especially relevant to the study. The second measure was cumulative in nature, with participants' selfreported proficiencies understanding, speaking, and reading averaged together for an aggregate proficiency score. Participants whose proficiency in English and in Mandarin did not differ by more than 1 point on the zero-to-five Likert scale were rated as equally proficient in both languages. Participants whose Mandarin proficiency exceeded English proficiency by more than 1 point were included in a higher-L1 proficiency group. No participants reported higher L2 than L1 proficiency. Both measures yielded comparable sample sizes across groups, although the exact group membership varied.

\section{RESULTS}

To test the predictions that task, language of encoding, and language of retrieval influence accessibility of everyday knowledge, we performed $3 \times 2 \times 2$ ANOVAs both by subjects and by items. Results are reported in Table 1 and reveal significant main effects of task, encoding language, and retrieval language on both accuracy and speed of response, as well as significant three-way and two-way interactions in the accuracy analyses.

To test the hypothesis that different types of knowledge are differentially susceptible to language-dependent memory, we performed separate analyses on multivalent, bivalent, and univalent responses. Planned follow-up $t$ tests to the three-way interaction were performed correcting for multiple comparisons using the Bonferroni method (Howell, 1995; Neter, Wasserman, \& Kutner, 1990) and based on the mean square error of the omnibus ANOVA.

In the multivalent task, the accessibility of responses increased when the same language was used at the time of encoding and retrieval (see Figure 1). This languagedependency effect was significant for each of the four responses, although its magnitude attenuated over time for speed of retrieval (see Table 2). Mandarin responses were significantly more likely to emerge during Mandarin interviews $(M=.76, S E=.03)$ than during English interviews $(M=.45, S E=.03)\left[t_{1}(19)=3.22, p<.05, \eta^{2}=\right.$ $\left.0.62 ; t_{2}(7)=4.29, p<.01, \eta^{2}=.87\right]$. English responses were significantly more likely to emerge during English interviews $(M=.41, S E=.02)$ than during Mandarin interviews $(M=.16, S E=.03)\left[t_{1}(19)=2.92, p<.05\right.$, $\left.\eta^{2}=.62 ; t_{2}(7)=3.87, p<.01, \eta^{2}=.84\right]$. For speed of memory retrieval, the effect of language match was significant only for information encoded in Mandarin, with faster retrieval in Mandarin $(M=10.47, S E=.97)$ than in English $(M=14.31, S E=1.44)\left[t_{1}(19)=3.22, p<.01\right.$, $\left.\eta^{2}=.31 ; t_{2}(7)=3.06, p<.05, \eta^{2}=.86\right]$.

In the bivalent task (see Figure 2), results revealed that the likelihood of drawing from a particular body of knowledge and the speed of memory retrieval were languagedependent. Participants were more likely to access information encoded in Mandarin when interviewed in Mandarin $(M=.72, S E=.06)$ than in English $(M=$ $.49, S E=.07)\left[t_{1}(19)=3.01, p<.05, \eta^{2}=.76 ; t_{2}(7)=\right.$ $\left.6.25, p<.001, \eta^{2}=.71\right]$ and were more likely to access information encoded in English when interviewed in English $(M=.51, S E=.07)$ than in Mandarin $(M=.28$, $S E=.06)\left[t_{1}(19)=3.01, p<.05, \eta^{2}=.18 ; t_{2}(7)=2.76\right.$, $\left.p=.06, \eta^{2}=.41\right]$. For speed of memory retrieval, the effect of language match was significant only for information encoded in Mandarin [Mandarin retrieval $M=$ 3.62, $S E=0.49$; English retrieval $M=7.49, S E=1.21$; $\left.t_{1}(18)=4.92, p<.01 ; t_{2}(7)=2.77, p=.08\right]$, confirming a stronger language-dependent memory effect in a more proficient language.

In the univalent task (see Figure 3), although accuracy results did not reveal language-dependent memory $(p>$ $.05)$, reaction times followed the patterns observed for bivalent and multivalent knowledge. Memories encoded

Table 1

Results of $2 \times 2 \times 3$ ANOVAs for Accuracy and Reaction Time

\begin{tabular}{|c|c|c|c|c|c|c|}
\hline Effect & $\begin{array}{c}F \text { Test } \\
\text { (by participants and by items) }\end{array}$ & $\begin{array}{c}\text { Effect } \\
\text { Size }\left(\eta^{2}\right)\end{array}$ & $M S_{\mathrm{e}}$ & Condition & $M$ & $S E$ \\
\hline \multicolumn{7}{|c|}{ Proportion Correct } \\
\hline \multirow[t]{2}{*}{ Encoding language (EL) } & $F_{1}(1,19)=46.03, p<.001$ & .71 & .09 & Mandarin: & .67 & .02 \\
\hline & $F_{2}(1,21)=7.62, p<.05$ & .27 & .05 & English: & .42 & .02 \\
\hline \multirow[t]{2}{*}{ Retrieval language (RL) } & $F_{1}(1,19)=18.51, p<.001$ & .49 & .01 & Mandarin: & .59 & .01 \\
\hline & $F_{2}(1,21)=0.96, p=.34$ & .05 & .02 & English: & .53 & .01 \\
\hline \multirow[t]{3}{*}{ Task } & $F_{1}(2,38)=177.49, p<.001$ & .90 & .02 & Multivalent: & .44 & .01 \\
\hline & $F_{2}(2,21)=95.76, p<.001$ & .90 & .02 & Bivalent: & .50 & .0001 \\
\hline & & & & Univalent: & .73 & .02 \\
\hline \multirow[t]{2}{*}{$\mathrm{RL} \times \operatorname{task}$} & $F_{1}(2,38)=16.87, p<.01$ & .47 & .02 & & & \\
\hline & $F_{2}(2,21)=4.82, p<.05$ & .32 & .02 & & & \\
\hline \multirow[t]{2}{*}{$\mathrm{EL} \times \mathrm{RL}$} & $F_{1}(1,19)=6.20, p<.05$ & .25 & .12 & & & \\
\hline & $F_{2}(1,21)=42.79, p<.001$ & .67 & .01 & & & \\
\hline \multirow[t]{2}{*}{$\mathrm{EL} \times \mathrm{RL} \times$ task } & $F_{1}(2,38)=50.67, p<.001$ & .73 & .41 & & & \\
\hline & $F_{2}(2,21)=8.12, p<.01$ & .44 & .01 & & & \\
\hline \multicolumn{7}{|c|}{ Reaction Times } \\
\hline \multirow[t]{2}{*}{ EL } & $F_{1}(1,10)=13.55, p<.01$ & .56 & 2.42 & Mandarin: & 6.58 & 0.62 \\
\hline & $F_{2}(1,20)=5.22, p<.05$ & .21 & 17.17 & English: & 5.59 & 0.58 \\
\hline \multirow[t]{2}{*}{ RL } & $F_{1}(1,10)=12.32, p<.01$ & .55 & 17.28 & Mandarin: & 4.81 & 0.66 \\
\hline & $F_{2}(1,20)=4.71, p<.05$ & .19 & 7.23 & English: & 7.35 & 0.71 \\
\hline \multirow[t]{3}{*}{ Task } & $F_{1}(2,20)=112.85, p<.001$ & .92 & 7.97 & Multivalent: & 10.0 & 0.91 \\
\hline & $F_{2}(2,20)=22.28, p<.001$ & .69 & 24.22 & Bivalent: & 4.65 & 0.68 \\
\hline & & & & Univalent: & 3.61 & 0.51 \\
\hline
\end{tabular}


A
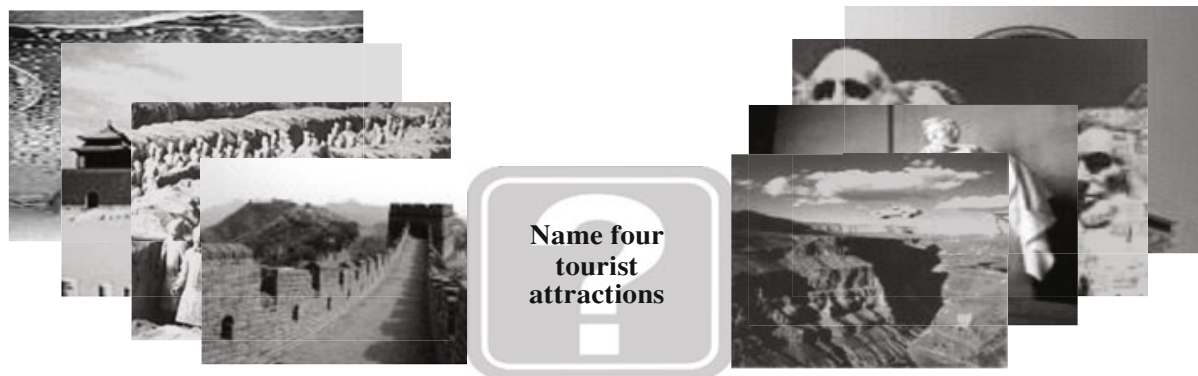

B

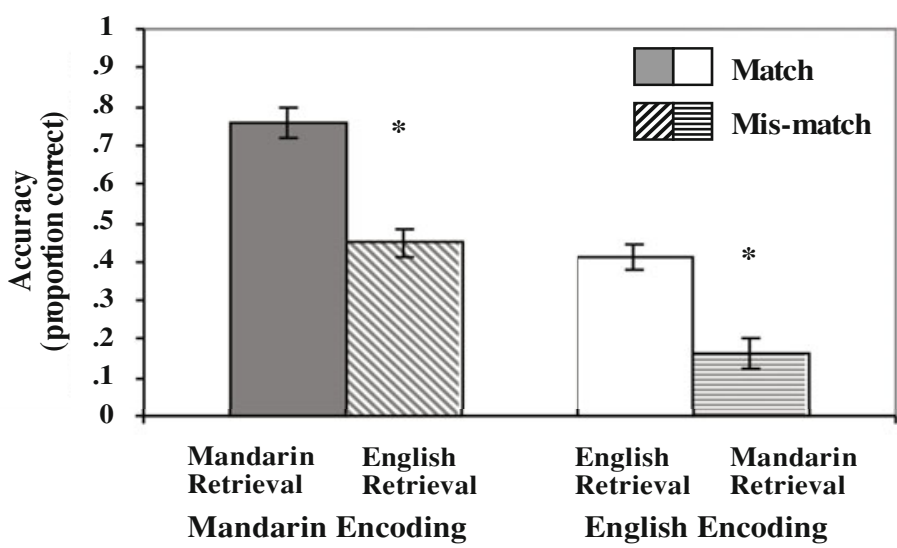

$\mathbf{C}$

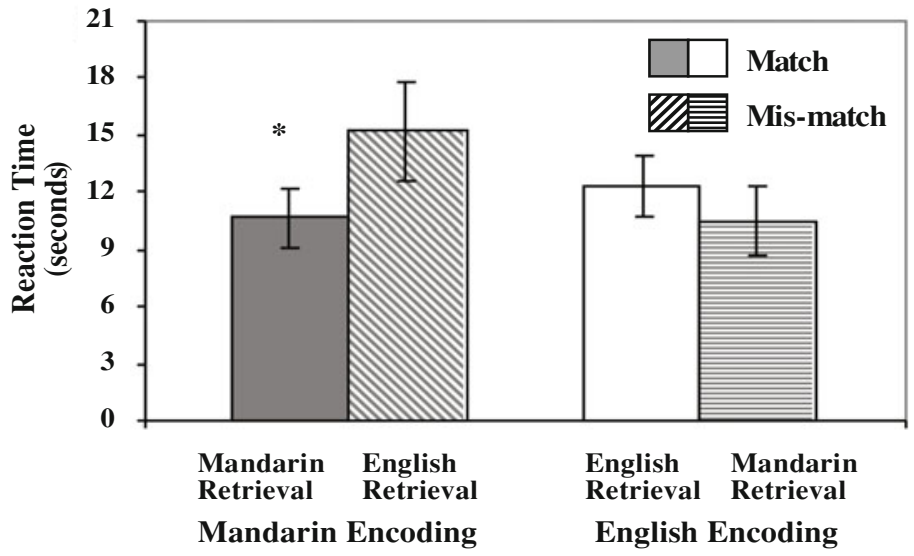

Figure 1. Language-dependent memory effects in the multivalent task. Panel A shows an example of a prompt. Participants were required to provide four responses, some of which may have been learned in Mandarin (e.g., the Great Wall of China, Silk Road, Terra-Cotta Army, the Forbidden City) and others in English (e.g., the Grand Canyon, Lincoln Memorial, Mount Rushmore, the Seattle Needle). Panel B shows proportions of questions answered correctly, and panel $\mathrm{C}$ shows reaction time results (means and standard errors, averaged across participants and responses) in each of the four encoding-by-retrieval conditions. ${ }^{*} p<.05$.

in Mandarin were accessed faster when the languages of encoding and retrieval matched $(M=3.66, S E=0.54)$ than when they were mismatched $(M=5.62, S E=0.69)$. Although this difference did not reach significance when the mean square error from the omnibus ANOVA was used (all $p \mathrm{~s}>.05$ ), it was significant both by subjects and by items when task-specific mean square error values were used in a priori ANOVAs (as described in Tabachnick \& Fidell, 2001) $\left[F_{1}(1,19)=5.60, p<.05, \eta^{2}=.25\right.$;
$\left.F_{2}(1,7)=14.05, p<.01, \eta^{2}=.67\right]$. This suggests that language-dependent memory effects in the univalent task were weaker than for other forms of knowledge.

Finally, to explore the role of proficiency in languagedependent memory, we performed two sets of additional analyses using relative proficiency in the two languages as a between-subjects independent variable (one with proficiency speaking and one with average proficiency understanding, speaking, and reading). The results confirmed 
A
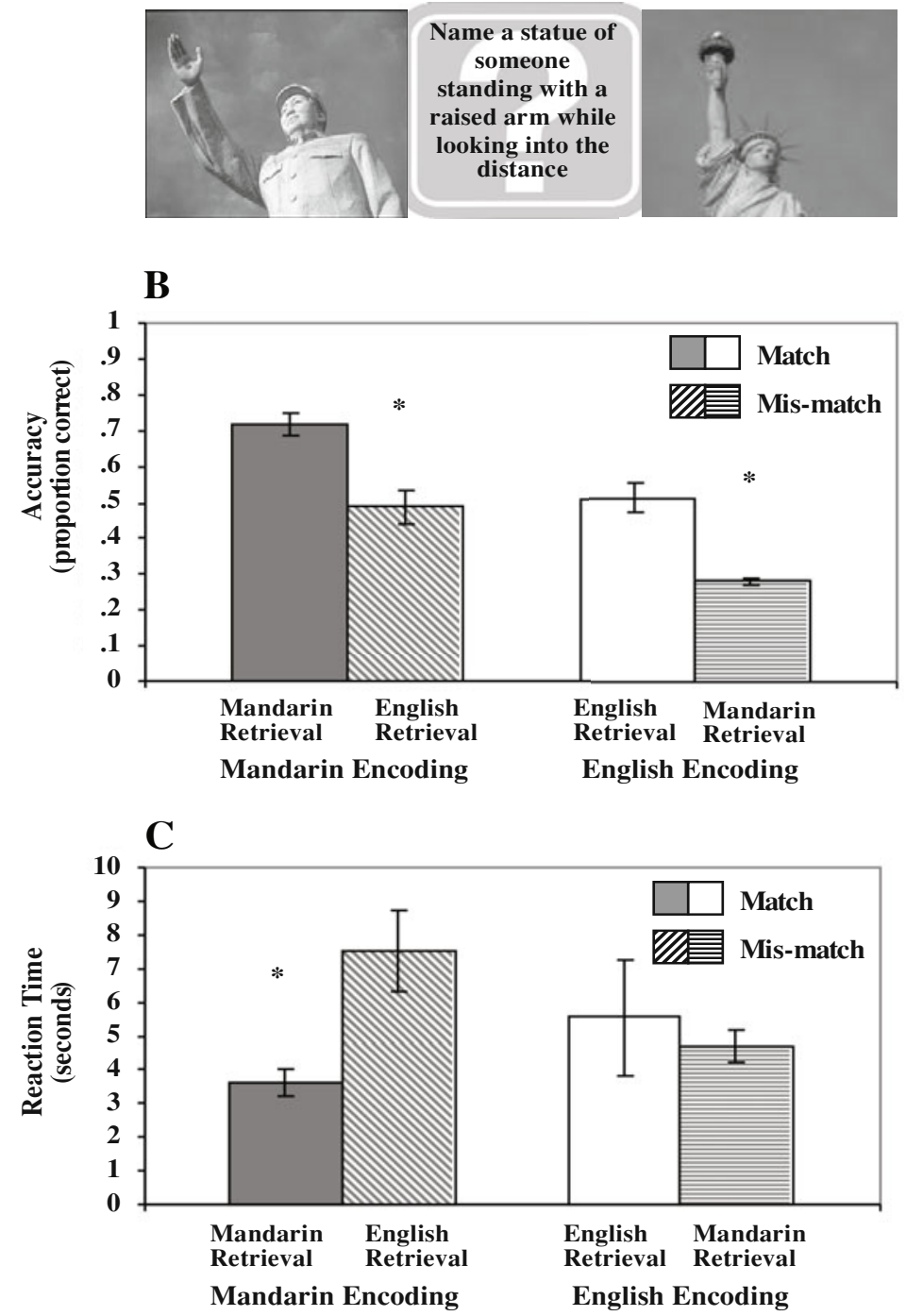

Figure 2. Language-dependent memory effects in the bivalent task. Panel A provides an example of a bivalent question and two possible answers (Chairman Mao, Statue of Liberty), panel B shows proportions of questions answered correctly, and panel $C$ shows reaction time results (means and standard errors) in each of the four encoding-by-retrieval conditions. ${ }^{*} p<.05$.

all main effects and interactions for both accuracy and speed of retrieval. No significant main effects or interactions with proficiency were observed in either of the two sets of proficiency analyses (all $p \mathrm{~s}>.1$ ), suggesting that language influenced retrieval of everyday knowledge similarly across proficiency groups.

\section{DISCUSSION}

In sum, across languages and tasks, general knowledge acquired in a particular language was more likely to be accessed when the same language was used at the time of recall. The magnitude of language-dependent memory was influenced by task type and language proficiency.
A comparison of effect sizes suggests that languagedependency was strong for multivalent $\left(\eta^{2}=.80\right)$ and bivalent $\left(\eta^{2}=.66\right)$ tasks and weak for the univalent task $\left(\eta^{2}=.04\right)$. Speed of memory retrieval was also facilitated by a match between languages of encoding and recall, and the magnitude of facilitation was strong for multivalent $\left(\eta^{2}=.44\right)$, bivalent $\left(\eta^{2}=.43\right)$, and univalent $\left(\eta^{2}=.21\right)$ tasks. These findings suggest that different types of knowledge are differentially sensitive to language-dependent memory, with language dependent effects more likely when multiple alternatives are available for retrieval. This difference among tasks may be localized to a selection mechanism, where whenever more than one correct answer is available, the selection mecha- 
Table 2

Response Data for Each Answer in the Multivalent Task

\begin{tabular}{|c|c|c|c|c|c|c|c|c|c|c|}
\hline \multirow{4}{*}{$\begin{array}{l}\text { Encoding } \\
\text { Language }\end{array}$} & \multicolumn{5}{|c|}{ Proportion Correct } & \multicolumn{5}{|c|}{ Reaction Times } \\
\hline & \multicolumn{4}{|c|}{ Retrieval Language } & \multirow{3}{*}{$\begin{array}{l}t \text { Value and } \\
\text { Effect Size }\end{array}$} & \multicolumn{4}{|c|}{ Retrieval Language } & \multirow{3}{*}{$\begin{array}{l}t \text { Value and } \\
\text { Effect Size }\end{array}$} \\
\hline & \multicolumn{2}{|c|}{ Mandarin } & \multicolumn{2}{|c|}{ English } & & \multicolumn{2}{|c|}{ Mandarin } & \multicolumn{2}{|c|}{ English } & \\
\hline & $M$ & $S E$ & $M$ & $S E$ & & $M$ & $S E$ & $M$ & $S E$ & \\
\hline \multicolumn{11}{|c|}{ First Response } \\
\hline Mandarin & .77 & .04 & .44 & .04 & $\begin{array}{l}t(19)=5.70 \\
\eta_{\mathrm{p}}^{2}=.63^{* *}\end{array}$ & 5.03 & 0.56 & 8.84 & 1.17 & $\begin{array}{l}t(19)=3.87 \\
\eta_{\mathrm{p}}^{2}=.44^{* *}\end{array}$ \\
\hline English & .19 & .03 & .44 & .04 & $\begin{array}{l}t(19)=4.87 \\
\eta_{\mathrm{p}}^{2}=.56^{* *}\end{array}$ & 4.27 & 0.96 & 4.17 & 0.50 & $\begin{array}{l}t(19)=0.09 \\
\eta_{\mathrm{p}}^{2}=.001\end{array}$ \\
\hline \multicolumn{11}{|c|}{ Second Response } \\
\hline Mandarin & .77 & .04 & .44 & .04 & $\begin{array}{l}t(19)=7.27 \\
\eta_{\mathrm{p}}^{2}=.74^{* *}\end{array}$ & 9.26 & 1.13 & 13.33 & 1.70 & $\begin{array}{l}t(19)=2.60 \\
\eta_{\mathrm{p}}^{2}=.26^{*}\end{array}$ \\
\hline English & .18 & .04 & .46 & .02 & $\begin{array}{l}t(19)=6.79 \\
\eta_{\mathrm{p}}^{2}=.71^{* *}\end{array}$ & 8.15 & 1.69 & 9.00 & 0.93 & $\begin{array}{l}t(19)=0.52 \\
\eta_{\mathrm{p}}^{2}=.02\end{array}$ \\
\hline \multicolumn{11}{|c|}{ Third Response } \\
\hline Mandarin & .77 & .04 & .49 & .05 & $\begin{array}{l}t(19)=6.34 \\
\eta_{\mathrm{p}}^{2}=.68^{* *}\end{array}$ & 12.64 & 1.20 & 17.27 & 3.00 & $\begin{array}{l}t(19)=5.50 \\
\eta_{\mathrm{p}}^{2}=.13^{*}\end{array}$ \\
\hline English & .16 & .03 & .37 & .04 & $\begin{array}{l}t(19)=6.24 \\
\eta_{\mathrm{p}}^{2}=.67^{* *}\end{array}$ & 12.14 & 1.83 & 13.15 & 1.92 & $\begin{array}{l}t(19)=0.49 \\
\eta_{\mathrm{p}}^{2}=.02\end{array}$ \\
\hline \multicolumn{11}{|c|}{ Fourth Response } \\
\hline Mandarin & .73 & .05 & .41 & .03 & $\begin{array}{l}t(19)=7.11 \\
\eta_{\mathrm{p}}^{2}=.73^{* *}\end{array}$ & 15.56 & 1.28 & 18.32 & 1.07 & $\begin{array}{l}t(19)=2.26 \\
\eta_{\mathrm{p}}^{2}=.21^{*}\end{array}$ \\
\hline English & .13 & .02 & .38 & .03 & $\begin{array}{l}t(19)=7.43 \\
\eta_{\mathrm{p}}^{2}=.74^{* *}\end{array}$ & 15.89 & 1.96 & 19.87 & 2.58 & $\begin{array}{l}t(19)=1.93 \\
\eta_{\mathrm{p}}^{2}=.22\end{array}$ \\
\hline
\end{tabular}

nism relies on additional markings to choose the answer. Such an explanation is consistent with a connectionist model of memory retrieval. In the univalent task, this additional step of selecting among multiple candidates is absent, since there is only one correct choice possible.

In the present study, the role of language proficiency was examined both across languages (by comparing performance in L1 vs. L2) and across bilinguals (by comparing a group who was equally proficient in both languages to a group who was more proficient in one of the two languages). Across languages, the magnitude of language-dependent memory was stronger in the more proficient language. For speed of retrieval, differences between language match and language mismatch were observed only in bilinguals' more proficient language. For accuracy, while languagedependent recall was observed in both Mandarin and English, the effect was stronger for bivalent memories encoded in Mandarin $\left(\eta^{2}=.76\right)$ than for memories encoded in English $\left(\eta^{2}=.18\right)$. Weaker effects for information encoded in English are likely due to encoding patterns in the less proficient language, which can involve internal translations into the more proficient language (Dornic, 1978; Schrauf, 2002). The stronger language-dependent memory for information encoded in Mandarin may also be due to repeated previous rehearsal and more opportunities for reactivation of those memories as a result of earlier acquisition.

Across bilinguals, both groups showed similar patterns of language-dependent recall, likely because bilinguals in the two groups did not differ enough to yield detectable proficiency effects. The limited variability in proficiency across groups may account for the difference between this study and studies of autobiographical memory, where bilinguals who are more proficient in one of their languages are more likely to show language-dependent memory (e.g., Larsen et al., 2002; Matsumoto \& Stanny, 2006). Alternatively, language-dependent memory for general knowledge may be less susceptible to proficiency effects than autobiographical memory is. Future research will need to test bilinguals whose proficiency levels differ more drastically or who are more proficient in their second language. Future research may also examine other cognitive variables that could make individuals more or less prone to languagedependent memory. Together, results suggest that languagedependent memory was consistent across all bilinguals, but was stronger in the more proficient language.

Pervasiveness of language-dependency effects in bilingual memory across multiple studies, languages, and tasks suggests a common cognitive phenomenon. However, a potential confound in interpreting these findings in terms of memory structure stems from cultural frameswitching. Prior research suggests that cultures differ in self-construal and memory patterns (e.g., Leichtman, Wang, \& Pillemer, 2003), and that bicultural bilinguals possess a highly fluid self and can shift cultural values and attribution patterns, depending on immediate cultural environment (Benet-Martínez, Leu, Lee, \& Morris, 2002; Hong, Morris, Chiu, \& Benet-Martínez, 2000; RamirezEsparza, Gosling, Benet-Martinez, Potter, \& Pennebaker, 2006). Bond and Yang (1982) suggested that cultural frame-switching can be cued by use of a specific language. In the present study, participants were questioned separately about the language at encoding and about the 
$\mathbf{A}$

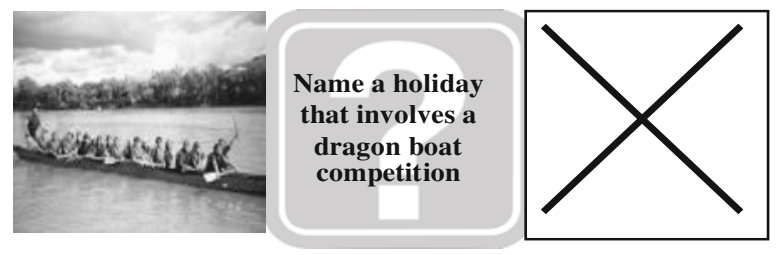

B
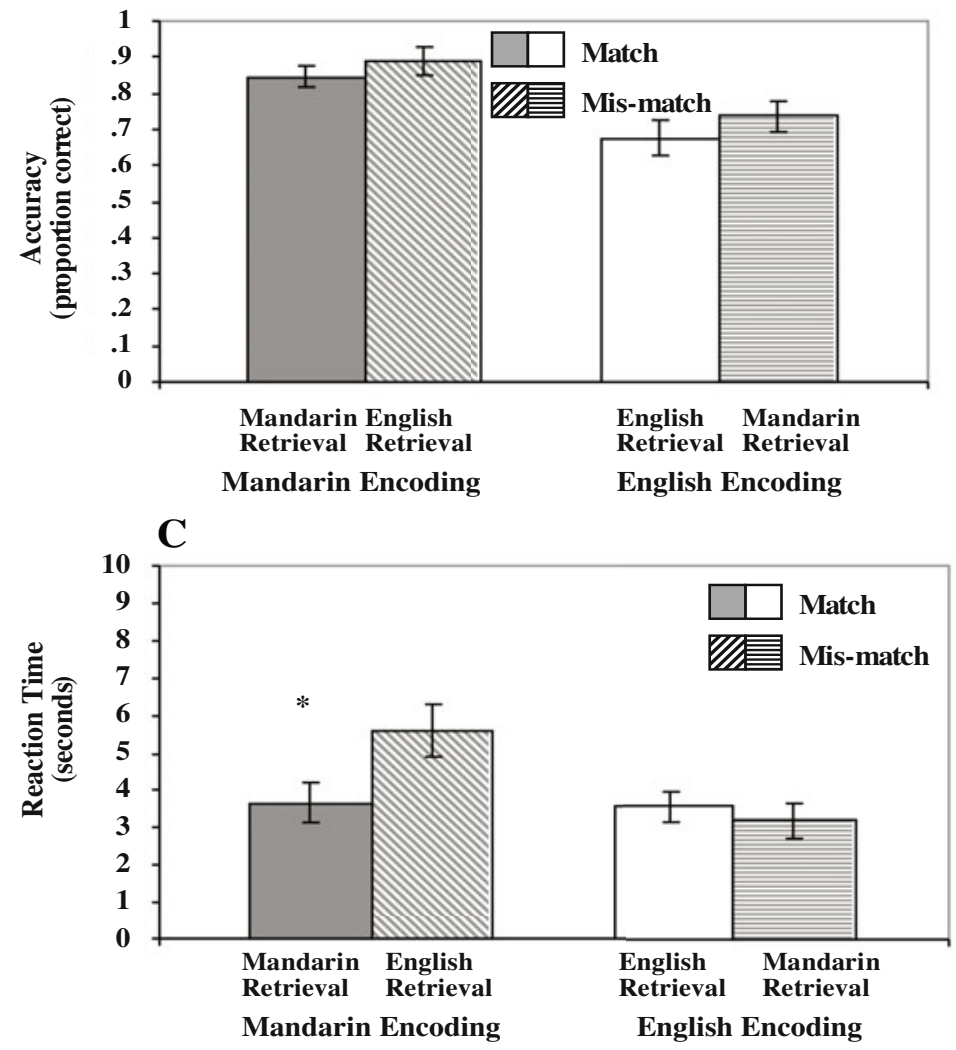

Figure 3. Language-dependent memory effects for the univalent task. Panel A provides an example of a univalent question and a single correct answer, likely learned in Mandarin. Panel B shows proportion of questions answered correctly, and panel $C$ shows the reaction time results (means and standard errors) in each of the four encoding-by-retrieval conditions. ${ }^{*} p<.05$.

culture at encoding for each response. The low number of instances where culture of encoding and language of encoding diverged did not permit statistical analyses separating the two. Future research may aim to increase the number of questions as well as to specifically construct questions that yield themselves better to separating the two (for example, by questioning bilinguals about an event such as watching a movie in English while situated in the Chinese culture).

Although it may appear to be a plausible contributor to the current findings, cultural frame-switching is an insufficient contributor for at least two reasons. First, Matsumoto and Stanny (2006) found language-dependent memory effects when the ambient language (experimenter's interview and bilingual's response) was constant and only the language of (culturally neutral) prompts varied. Second, semantic memory that is not culturally influenced or tied to the self, such as remembering random word lists and learning culturally neutral academic material shows comparable language-dependent memory effects. This suggests that, although cultural frame-switching may be a contributing and exacerbating factor in languagedependent memory, it is not likely to be a sufficient explanation for it.

More likely, the differential accessibility of long-term knowledge across languages is due to the existence of a general mechanism underlying interaction between memory and language. Such a mechanism may operate as part of a larger associative network, where language context and memory content are linked and where encoding con- 
text influences content retrieval. In addition, languagedependent memory may rely on language-specific principles that are not available to other types of context, such as thinking-for-speaking (Slobin, 2003), where thoughts are expressed in ways consistent with a particular language (Gentner \& Goldin-Meadow, 2003; Slobin, 1996). In this way, linguistic encoding of memory would etch languagespecific interpretation onto semantic content, rendering this content more accessible when the same language is used at the time of retrieval. Therefore, we propose that language-dependent memory is subject to both domaingeneral interactions between content and context, as well as to language-specific processes, such as linguistic effects on cognition.

Language-dependent memory may be characteristic of general human cognitive processing. Its functional benefit may have its roots in the increased likelihood that the same information would be needed when one is situated again in the same (linguistic) environment. Previous work by Rovee-Collier and colleagues suggests a developmental pattern where both content and context are initially processed together in the same manner and to the same degree (Rovee-Collier, Griesler, \& Earley, 1985; RoveeCollier \& Hayne, 1987; Rovee-Collier \& Shyi, 1992). For instance, very young infants are unable to separate target learning (e.g., kicking to activate a mobile) from incidental background (e.g., color of crib bumper) and show contextdependent memory, where memory for a target behavior is tied to memory for a background (e.g., will only kick to activate a mobile if the crib bumper is the same as at time of learning). Over the course of development, the ability to separate target from background and to distinguish relevant content from incidental context improves. However, it appears that, whereas a conscious distinction between content and context emerges, the cognitive system continues to register both, imprinting nontarget (linguistic) background onto memory content. These findings carry implications for applied settings in which accurate and efficient memory retrieval may be susceptible to linguistic influences, including legal practices (such as witness questioning and testimony), clinical intervention (such as accessibility of memories in therapy), and educational policy (such as academic assessment of multilingual students).

\section{AUTHOR NOTE}

This study was supported by Grants NICHD1R03HD046952-01A1 and NSFBCS-0418495 to the first author. We thank Richard Gerrig, Claudia Stanny, and two anonymous reviewers for helpful comments on this article; Dong Lu for assistance with data collection; Li Sheng for assistance with back translation; Tina Yao for reliability coding; and Caitlin Fausey and Henrike Blumenfeld for insightful discussions of this work. Correspondence regarding this article should be addressed to V. Marian, Department of Communication Sciences and Disorders, Northwestern University, Evanston, IL 60208-3570 (e-mail: v-marian@northwestern.edu).

\section{REFERENCES}

Baddeley, A. D. (1982). Your memory: A user's guide. New York: Macmillan.

Benet-Martínez, V., Leu, J., Lee, F., \& Morris, M. (2002). Negotiating biculturalism: Cultural frame-switching in biculturals with “oppositional” vs. "compatible" cultural identities. Journal of CrossCultural Psychology, 33, 492-516.

Bond, M. H., \& YANG, K. (1982). Ethnic affirmation versus crosscultural accommodation. Journal of Cross-Cultural Psychology, 13, 169-185.

Dalrymple-Alford, E. C., \& Aamiry, A. (1969). Language and category clustering in bilingual free recall. Journal of Verbal Learning \& Verbal Behavior, 8, 762-768.

Davies, G. M., \& Thomson, D. M. (Eds.) (1988). Memory in context: Context in memory. Chichester, U.K.: Wiley.

DoRnIC, S. (1978). The bilingual's performance: Language dominance, stress, and individual differences. In D. Gerver \& H. W. Sinaiko (Eds.), Language interpretation and communication (pp. 259-271). New York: Plenum.

DurgunoĞLu, A. Y., \& Roediger, H. L., III (1987). Test differences in accessing bilingual memory. Journal of Memory \& Language, 26, 377-391

Gentner, D., \& Goldin-Meadow, S. (2003). Language in mind: Advances in the study of language and thought. Cambridge, MA: MIT Press.

GopniK, A., \& Meltzoff, A. N. (1997). Words, thoughts, and theories. Cambridge, MA: MIT Press.

Gumperz, J. J., \& LEVInson, S. C. (EDS.) (1996). Rethinking linguistic relativity. Cambridge: Cambridge University Press.

Hong, Y., Morris, M. W., Chiu, C., \& Benet-Martínez, V. (2000). Multicultural minds: A dynamic constructivist approach to culture and cognition. American Psychologist, 55, 709-720.

Howell, D. C. (1995). Fundamental statistics for the behavioral sciences (3rd ed.). Belmont, CA: Duxbury Press.

KINTSCH, W. (1970). Recognition memory in bilingual subjects. Journal of Verbal Learning \& Verbal Behavior, 9, 405-409.

Koriat, A., \& Goldsmith, M. (1996). Memory metaphors and the real-life/laboratory controversy. Behavioral \& Brain Sciences, 19, 167-228.

Koven, M. (2001). Comparing bilinguals' quoted performance of self and others in telling the same experience in two languages. Language in Society, 30, 513-558.

Larsen, S., Schrauf, R. W., Fromholt, P., \& Rubin, D. C. (2002). Inner speech and bilingual autobiographical memory: A PolishDanish cross-cultural study. Memory, 10, 45-54.

Leichtman, M. D., Wang, Q., \& Pillemer, D. B. (2003). Cultural variations in interdependence and autobiographical memory: Lessons from Korea, China, India, and the United States. In R. Fivush \& C. A. Haden (Eds.), Autobiographical memory and the construction of self: Developmental and cultural perspectives (pp. 73-97). Mahwah, NJ: Erlbaum.

Marian, V., Blumenfeld, H. K., \& Kaushanskaya, M. (2007). The Language Experience and Proficiency Questionnaire (LEAP-Q): Assessing language profiles in bilinguals and multilinguals. Journal of Speech, Language, \& Hearing Research, 50, 940-967.

Marian, V., \& FAUSEY, C. M. (2006). Language-dependent memory in bilingual learning. Applied Cognitive Psychology, 20, 1-23.

Marian, V., \& Kaushanskaya, M. (2004). Language-mediated selfconstrual and emotion in bicultural bilinguals. Journal of Memory \& Language, 51, 190-201.

Marian, V., \& Neisser, U. (2000). Language-dependent recall of autobiographical memories. Journal of Experimental Psychology: General, 129, 361-368.

Matsumoto, A., \& Stanny, C. J. (2006). Language-dependent access to autobiographical memory in Japanese-English bilinguals and US monolinguals. Memory, 14, 378-390.

NeIsser, U. (1978). Memory: What are the important questions? In P. Grunenberg \& R. N. Sykes (Eds.), Practical aspects of memory (pp. 3-24). London: Academic Press.

Neter, J., Wasserman, W., \& Kutner, M. H. (1990). Applied linear statistical models (3rd ed.). Homewood, IL: Richard D. Irwin, Inc.

Ramirez-Esparza, N., Gosling, S. D., Benet-Martínez, V., Potter, J. P., \& Pennebaker, J. W. (2006). Do bilinguals have two personalities? A special case of cultural frame switching. Journal of Research in Personality, 40, 99-120.

Ross, M., Xun, W. Q. E., \& Wilson, A. E. (2002). Language and the bicultural self. Personality \& Social Psychology Bulletin, 28, $1040-1050$ 
Rovee-Collier, C., Griesler, P. C., \& Earley, L. A. (1985). Contextual determinants of retrieval in three-month-old infants. Learning \& Motivation, 16, 139-157.

Rovee-Collier, C., \& Hayne, H. (1987). Reactivation of infant memory. In H. Reese (Ed.), Advances in child development and behavior (pp. 185-238). New York: Academic Press.

Rovee-Collier, C., \& Shyi, G. (1992). A functional and cognitive analysis of infant retention. In C. Brainerd, M. Howe, \& V. Reyna (Eds.), Development of long-term retention (pp. 3-55). New York: Springer.

Schacter, D. L., \& Tulving, E. (1994). Memory systems. Cambridge, MA: MIT Press.

SCHRAUF, R. W. (2002). Bilingual inner speech as the medium of crossmodular retrieval in autobiographical memory. Behavioral \& Brain Sciences, 25, 698-699.

Schrauf, R. W., \& Rubin, D. C. (1998). Bilingual autobiographical memory in older adult immigrants: A test of cognitive explanations of the reminiscence bump and the linguistic encoding of memories. Journal of Memory \& Language, 39, 437-457.

SLOBIN, D. I. (1996). From "thought and language" to "thinking for speaking.” In J. J. Gumperz \& S. C. Levinson (Eds.), Rethinking linguistic relativity (pp. 70-96). Cambridge: Cambridge University Press.
Slobin, D. I. (2003). Language and thought online: Cognitive consequences of linguistic relativity. In D. Gentner \& S. Goldin-Meadow (Eds.), Language in mind: Advances in the study of language and thought (pp. 157-192). Cambridge, MA: MIT Press.

TABACHNICK, B. G., \& FidelL, L. S. (2001). Using multivariate statistics (4th ed.). Needham Heights, MA: Pearson Education.

Tulving, E., \& Thomson, D. (1973). Encoding specificity and retrieval processes in episodic memory. Psychological Review, 80, 352-373.

Waxman, S. R., \& Braun, I. E. (2005). Consistent (but not variable) names as invitations to form object categories: New evidence from 12-month-old infants. Cognition, 95, B59-B68.

\section{NOTE}

1. Most of the questions probing for material learned in English were specific to local Midwestern geopolitics, since general information about the United States may have been acquired in Chinese prior to arrival to the USA.

(Manuscript received August 2, 2006; revision accepted for publication January 2, 2007.) 